\title{
Análise tridimensional tomográfica das dimensões da cavidade nasal e do espaço aéreo nasal e faríngeo superior de indivíduos submetidos à expansão da maxila cirurgicamente assistida
}

\author{
Three-dimensional tomographic analysis of the dimensions of the nasal cavity and the nasal and \\ upper pharyngeal airway space of subjects undergoing surgically assisted maxillary expansion \\ Análisis tomográfico tridimensional de las dimensiones de la cavidad nasal y del espacio de las vías \\ respiratorias nasales y faríngeas superiores de individuos sometidos a expansión maxilar asistida \\ quirúrgicamente
}

Eduardo Stedile Fiamoncini ORCID: https://orcid.org/0000-0002-5283-4133 Clínica privada, Brasil

E-mail: eduardofiamoncini@alumni.usp.br Carolina Gachet Barbosa ORCID: https://orcid.org/0000-0001-9861-2037 Universidade de São Paulo, Brasil E-mail: carol.gachet@hotmail.com Isadora Molina Sanches ORCID: https://orcid.org/0000-0002-0999-2523 Universidade de São Paulo, Brasil

E-mail: isadoramolinasanches@gmail.com Eduardo Sanches Gonçales ORCID: https://orcid.org/0000-0002-6682-7006 Universidade de São Paulo, Brasil E-mail: eduardogoncales@usp.br

\begin{abstract}
Resumo
A expansão da maxila cirurgicamente assistida (EMCA) tem como principal objetivo o aumento da dimensão transversal da arcada dentária superior em indivíduos com atresia maxilar. Seus efeitos não são restritos à maxila, atuando também sobre a cavidade nasal e espaço aéreo faríngeo. O presente estudo teve o objetivo de avaliar a ocorrência de alterações dimensionais da cavidade nasal e no espaço aéreo nasal e faríngeo superior em indivíduos submetidos a expansão maxilar cirurgicamente assistida, por meio de 2 osteotomias diferentes. As estruturas do espaço aéreo nasal e faríngeo foram analisadas por meio de tomografias computadorizadas de feixe cônico pré e pósoperatórias de 29 indivíduos adultos, submetidos à expansão de maxila cirurgicamente assistida. Mensuração volumétrica do espaço aéreo nasal e faríngeo superior e medidas lineares das dimensões da cavidade nasal foram realizadas por meio do uso do software Dolphin Imaging 11,7. Os dados obtidos foram tabulados e submetidos à análise estatística pelos testes de variância ANOVA e Tukey $(\mathrm{p}<0,05)$. Os resultados mostraram que ambas as osteotomias foram eficazes na correção da deficiência transversal da maxila e apresentaram efeitos semelhantes sobre a cavidade nasal e o espaço aéreo faríngeo. Foi verificado aumento de volume do espaço aéreo faríngeo, também na região da hipofaringe. Além disso, observou-se que a EMCA promove um aumento na largura do assoalho da cavidade nasal após a expansão, e, consequentemente aumento no volume do espaço aéreo nasal.
\end{abstract}

Palavras-chave: Cavidade nasal; Técnica de expansão palatina; Tomografia computadorizada por Raios X.

\begin{abstract}
The main objective of the surgically assisted rapid maxillary expansion (SARME) is to increase the transversal dimension of the upper dental arch in individuals with maxillary atresia. Its effects are not restricted to the maxilla, also acting on the nasal cavity and pharyngeal airway. The present study aimed to evaluate the occurrence of dimensional changes in the nasal cavity and nasal and upper pharyngeal airway space in individuals submitted to surgically assisted maxillary expansion using 2 different osteotomies. Nasal and pharyngeal airway space structures were analyzed by pre-and post-operative cone beam computed tomography scans of 29 adult subjects submitted to surgically assisted maxillary expansion. Volumetric measurement of the nasal and upper pharyngeal airway and linear measurements of the nasal cavity were performed using Dolphin Imaging 11,7 software. The data were tabulated and
\end{abstract}


submitted to statistical analysis by ANOVA analysis of variance and Tukey test $(\mathrm{p}<0,05)$. The results showed that both osteotomies were effective in correcting the transverse maxillary deficiency and presented similar effects on the nasal cavity and pharyngeal airway space. There was an increase in the volume of pharyngeal airway, also observed in the hypopharynx region. In addition, it was observed that SARME promotes an increase in the floor width of the nasal cavity after expansion, and, consequently, an increase in nasal airway volume.

Keywords: Nasal cavity; Palatal expansion technique; Tomography, X-Ray computed.

\section{Resumen}

La expansión maxilar asistida quirúrgicamente (SCME) tiene como objetivo aumentar la dimensión transversal de la arcada superior en individuos con atresia maxilar. Sus efectos no se limitan al maxilar, sino que también afectan a la cavidad nasal y al espacio de las vías respiratorias faríngeas. El propósito de este estudio fue evaluar la aparición de cambios dimensionales en la cavidad nasal, el espacio de las vías respiratorias nasales y el espacio de las vías respiratorias faríngeas superiores en individuos que se sometieron a una expansión maxilar asistida quirúrgicamente mediante dos osteotomías diferentes. Las estructuras del espacio de las vías respiratorias nasales y faríngeas se analizaron mediante tomografías computarizadas (TC) de haz cónico antes y después de la operación. Medidas postoperatorias de 29 sujetos adultos sometidos a una expansión maxilar asistida quirúrgicamente. Medición volumétrica del espacio de las vías respiratorias nasales y las mediciones de la parte superior de la faringe y las mediciones lineales de las dimensiones de la cavidad nasal se realizaron con el software Dolphin Imaging 11.7. Los datos se tabularon y se analizaron estadísticamente mediante las pruebas de varianza ANOVA y Tukey $(\mathrm{p}<0,05)$. Los resultados mostraron que ambas osteotomías eran eficaces para corregir la deficiencia transversal del maxilar y tenían efectos similares en la cavidad nasal y el espacio de las vías respiratorias faríngeas. También se observó un aumento del volumen del espacio faríngeo en la región hipofaríngea. Además, se ha observado que la ACME favorece el aumento de la anchura del suelo de la cavidad nasal tras la expansión, y, consiguiente aumento del volumen del espacio aéreo nasal.

Palabras clave: Cavidad nasal; Técnica de expansión palatina; Tomografía computarizada por Rayos X.

\section{Introdução}

A deficiência transversal da maxila, ou discrepância maxilar transversal, é uma alteração esquelética caracterizada por palato ogival, apinhamento dentário, rotação dental e mordida cruzada posterior uni ou bilateral (Gonçales, 2010; Oliveira, 2016). Esses indivíduos frequentemente apresentam um estreitamento da cavidade nasal, podendo resultar em aumento da resistência ao fluxo de ar nasal e prejuízo respiratório (Gungor \& Turkkahraman, 2009; Gonçales, 2010; Oliveira, 2016).

A opção pelo tratamento deve considerar, durante a fase diagnóstica, a magnitude da discrepância, origem esquelética e/ou dentária, a idade do paciente e a associação com outros tipos de deformidades craniofaciais (Silva Filho et al., 1989). O tratamento da deficiência transversal da maxila pode ser realizado por meio de procedimentos ortopédicos nos indivíduos em fase de crescimento, associado ao tratamento cirúrgico em indivíduos com o crescimento ósseo completo (Verstraaten et al., 2010).

A expansão da maxila é um procedimento rotineiramente utilizado por ortodontistas para o tratamento da mordida cruzada posterior (Capelozza \& Silva, 1997). A mesma técnica tem sido associada à expansão da cavidade nasal e melhora da respiração em pacientes com desordens respiratórias por constrição nasal, aliviando os sintomas e minimizando a respiração bucal (McNamara et al., 2015). Isso se deve ao fato de que a expansão da maxila possivelmente resultará em aumento do diâmetro transversal da maxila e alargamento do assoalho nasal, aumentando a permeabilidade das vias aéreas (Bell \& Epker, 1976).

A expansão da maxila cirurgicamente assistida (EMCA) resulta em um discreto aumento na área da cavidade nasal, alargamento da abertura piriforme na porção basal e aumento simétrico na distância da base da abertura piriforme ao septo nasal bilateralmente (Gonçales et al., 2007), sendo esses mesmos efeitos observados nos indivíduos submetidos a tratamento ortopédico ou cirúrgico (Altug-Atac et al., 2010).

Em consequência ao seu impacto na via aérea nasal, a EMCA pode ser potencialmente utilizada com uma opção de tratamento para constrição nasal em pacientes com deficiência maxilar transversa associada. Entretanto, as alterações do 
espaço aéreo nasal não podem ser completamente definidas, principalmente devido às inconsistências na metodologia dos trabalhos presentes na literatura e acompanhamento insuficiente. (Buck et al., 2016)

O presente estudo teve como principal objetivo avaliar, por meio da análise de tomografias computadorizadas de feixe cônico, as possíveis alterações dimensionais no espaço aéreo faríngeo e cavidade nasal em indivíduos submetidos à expansão de maxila cirurgicamente assistida. Além de comparar as alterações dimensionais volumétricas do espaço aéreo e lineares da cavidade nasal entre os desenhos da osteotomia lateral da maxila com degrau no pilar zigomático e linear.

\section{Metodologia}

Avaliou-se os exames de tomografia computadorizada de feixe cônico (TCFC) de indivíduos adultos, diagnosticados com discrepância transversa de maxila e submetidos à EMCA. As tomografias foram realizadas em 03 tempos distintos, sendo eles o pré-operatório (T1), após cessada a ativação do aparelho expansor (T2) e no pós-operatório de 180 dias (T3). Os exames de TCFC foram realizados no protocolo de aquisição estendido, com Voxel de 0,3mm e 28,45mA, no aparelho I-Cat Classic ${ }^{\circledR}$ (Imaging Sciences, Hatfield, Pensilvânia - EUA).

Foram excluídos exames de indivíduos que apresentavam fissuras palatinas, síndromes craniofaciais ou lesões ósseas na área de interesse do estudo. Ainda, foram excluídos exames de indivíduos em fase de crescimento, ou que não realizaram os exames de controle dentro dos tempos propostos. No total foram avaliados 29 indivíduos, divididos em dois grupos, de acordo com a técnica cirúrgica utilizada.

No Grupo I, a técnica cirúrgica escolhida foi a osteotomia Le Fort I subtotal, com degrau no pilar zigomático, assim como proposto por Betts et al. (1995), porém sem a separação do septo nasal. Todos os pacientes tiveram um expansor palatino instalado no pré-operatório, sendo que 8 pacientes receberam expansor do tipo Hyrax e 6 pacientes receberam expansor do tipo Hass fixado aos pré-molares e primeiros molares maxilares.

A ativação foi iniciada 5 dias após o procedimento cirúrgico, em que o aparelho foi ativado um quarto de volta $(0,25$ $\mathrm{mm}$ ), 2 vezes ao dia (a cada $12 \mathrm{~h}$ ), resultando em $0,5 \mathrm{~mm}$ de expansão a cada $24 \mathrm{~h}$, durante 7 dias. Nos 7 dias seguintes os pacientes foram orientados a não fazer nenhum tipo de ativação, intercalando semanas de ativação e semanas de descanso até que as cúspides palatinas dos molares e pré-molares superiores estivessem em contato com as vertentes triturantes das cúspides vestibulares dos dentes inferiores posteriores, obtendo a expansão necessária com uma ligeira sobre correção. Após atingir a expansão desejada, o aparelho foi estabilizado e mantido por cerca de 4 a 6 meses, a critério do ortodontista.

O Grupo II foi submetido à osteotomia linear proposta por Kraut (1984), acrescentada da osteotomia da sutura pterigopalatina e sem separação do septo nasal. Os participantes utilizaram expansor palatino do tipo Hyrax fixado aos prémolares e primeiros molares maxilares. A ativação foi iniciada 7 dias após o procedimento cirúrgico, a qual foi composta por: um quarto de volta $(0,25 \mathrm{~mm}), 3$ vezes ao dia (a cada $8 \mathrm{~h}$ ), resultando em $0,75 \mathrm{~mm}$ de expansão a cada $24 \mathrm{~h}$. A expansão necessária era obtida com uma ligeira sobre correção em média após 14,4 dias. Após a expansão desejada, o aparelho foi estabilizado e mantido por mais 4 meses.

As imagens foram avaliadas por meio do programa Dolphin Imaging (versão 11.7, Management Solutions, Chatsworth, Califórnia, EUA), utilizando ferramentas específicas do programa, com a qual foi realizada a análise volumétrica e linear da cavidade nasal e a posição do septo. As mesmas foram orientadas de maneira padronizada, definindo a posição da imagem nos planos anatômicos de acordo com pontos cefalométricos pré-estabelecidos (Figura 1). 
Figura 1: A análise volumétrica $\left(\mathrm{mm}^{3}\right)$ da cavidade aérea nasal foi realizada utilizando o software Dolphin Imaging (versão 11,7, Management Solutions, Chatsworth, Califórnia, EUA), delimitada de acordo com pontos cefalométricos prédeterminados.

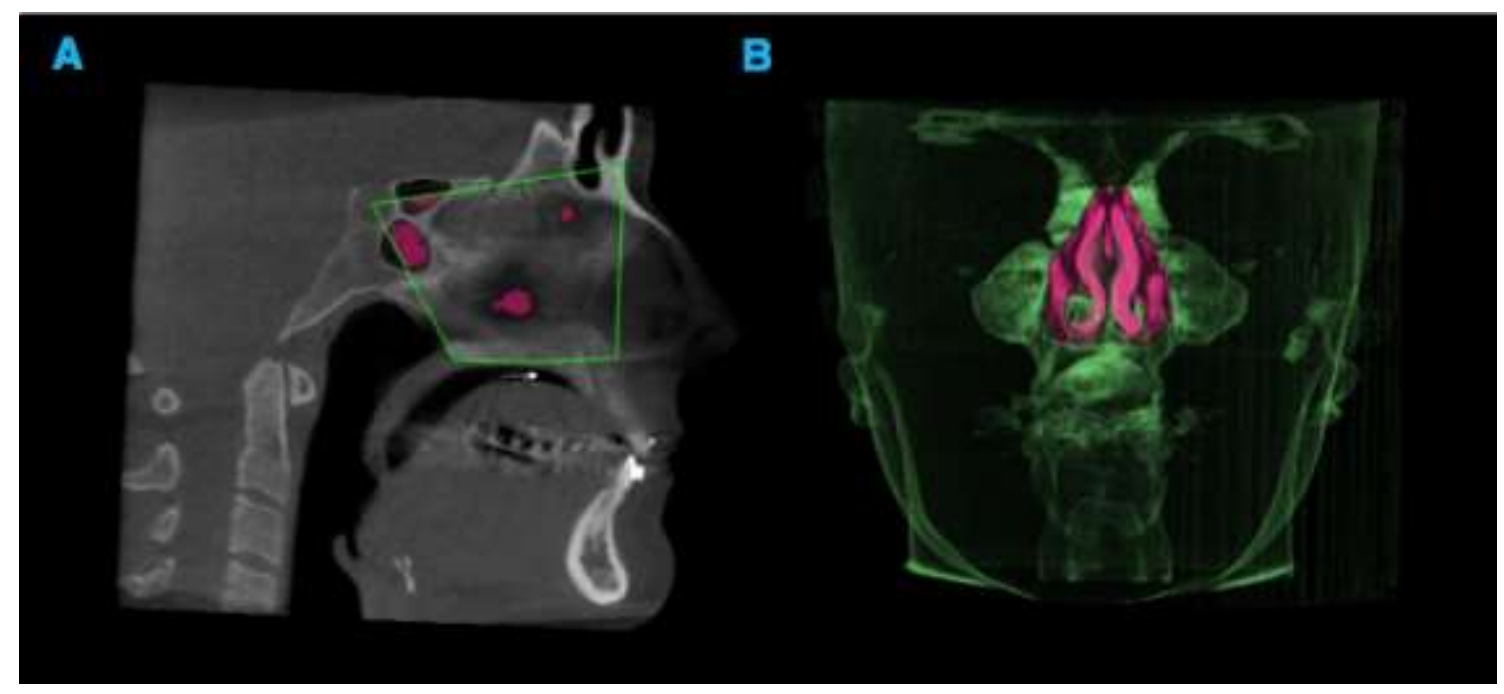

Fonte: Autores (2021).

Para a análise linear $(\mathrm{mm})$ das imagens dos exames de TCFC, as medidas foram realizadas em diferentes pontos verticais e anteroposteriores. Para o posicionamento vertical das medidas (realizadas em três planos), utilizou-se a medida do comprimento vertical do septo nasal, desde sua porção mais superior (região de inserção no etmóide) até sua porção mais inferior (região de articulação com o assoalho da cavidade nasal).

Três pontos equidistantes foram determinados ao longo da medida vertical do septo e os locais das medidas foram dispostos de inferior para superior, a partir do assoalho nasal, dividindo a cavidade nasal em compartimentos distintos (inferior, médio e superior). Ainda, as mensurações verticais foram realizadas em três níveis horizontais distintos, posicionados em uma reformatação axial com base da coroa de dentes maxilares, sendo eles: anterior (caninos), média (segundos prémolares) e posterior (segundos molares) (Figura 2).

A metodologia para a realização medidas lineares horizontais da cavidade e do septo nasal foi adaptada da metodologia utilizada por Gonçales et al. (2007), e as mensurações foram realizadas utilizando a ferramenta digitalize/measure, em reformatações coronais:

a. Largura da base da abertura piriforme (LBAP);

b. Largura mediana da abertura piriforme (LMAP);

C. Largura superior da abertura piriforme (LSAP);

a. Distância da base da parede lateral da abertura piriforme ao septo do lado direito (BAPSD); Distância da base da parede lateral da abertura piriforme ao septo do lado esquerdo (BAPSE);

b. Largura mediana da parede lateral da abertura piriforme ao septo do lado direito (LMAPSD); Largura mediana da parede lateral da abertura piriforme ao septo do lado esquerdo (LMAPSE);

C. Largura superior da parede lateral da abertura piriforme ao septo do lado direito (LSAPSD); Largura superior da parede lateral da abertura piriforme ao septo do lado esquerdo (LSAPSE). 
Figura 2. Ilustração das mensurações lineares $(\mathrm{mm})$ látero-laterais da cavidade nasal e posição do septo em reformatações coronais nas regiões: A) Anterior (13 e 23); B) Média (15 e 25) e C); Posterior (17 e 27).
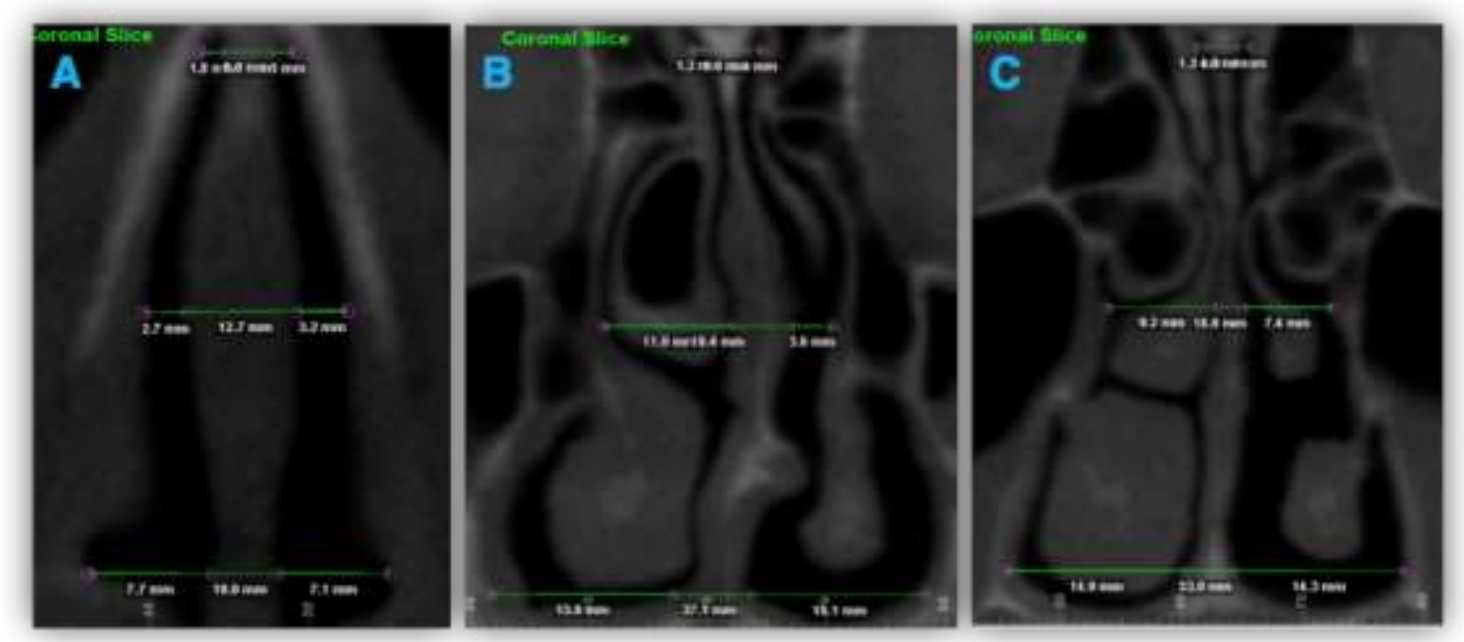

Fonte: Autores (2021).

A análise estatística foi realizada com o software Statistica 12 (Dell Inc., Round Rock, Texas, EUA), considerando o nível de significância de 5\% ( < .05). A reprodutibilidade do método foi determinada utilizando 30 exames de TCFC selecionados aleatoriamente e avaliados pelo mesmo examinador com o intervalo de trinta dias. O teste Dahlberg foi usado para determinação da concordância intra-examinador das várias medidas.

Os três tempos cirúrgicos foram avaliados por meio do teste ANOVA. A avaliação foi composta por duas etapas: a) todos os indivíduos da amostra $(n=29)$, com o objetivo de analisar as alterações ocorridas durante o período da expansão entre cada um dos tempos operatórios avaliados e b) os grupos isolados (I e II), para avaliar as possíveis alterações ocorridas entre os tempos operatórios de acordo com cada técnica cirúrgica utilizada. As alterações estatisticamente significantes foram avaliadas por meio do teste post-hoc de Tukey para avaliar as diferenças entre os grupos e os tempos de tratamento.

\section{Resultados}

O grupo I foi composto de 14 indivíduos adultos ( 9 do gênero feminino e 5 do gênero masculino), com idade média de 25,3 anos (DP = 5,71 anos). O grupo II foi composto de 15 indivíduos adultos (9 do gênero feminino e 6 do gênero masculino), com idade média de 30,2 anos (DP = 7,4 anos). Os grupos foram avaliados com relação a alterações do volume da cavidade nasal, dimensões transversais da cavidade nasal e o posicionamento do septo.

$\mathrm{O}$ volume da cavidade nasal foi avaliado nos seguintes tempos: T1 x T2; T1 x T3; e T2 x T3. Evidenciando-se o aumento estatisticamente significante $(\mathrm{P}<.05)$, quando presente, das medidas volumétricas da dimensão da cavidade nasal em todos os pacientes da pesquisa $(n=29)$, no grupo I $(n=14)$ e grupo II $(n=15)$ (Tabela 1$)$. Ao avaliar o grupo I isoladamente, houve diferença estatisticamente significativa para o volume $(\mathrm{p}=0,045)$. 
Tabela 1. Medidas de volume $\left(\mathrm{mm}^{3}\right)$ da cavidade nasal nos tempos pré-operatório (T1), após cessada a ativação do aparelho (T2) e pós-operatório de 180 dias (T3). Médias (M), desvio padrão (dp), resultado do teste Anova para medidas repetidas (p) $(* \mathrm{p}<0,05)$.

\begin{tabular}{lcccc}
\hline Volume & T1 M(dp) & T2 M(dp) & T3 M(dp) & p \\
\hline Total & 17338,1 & $18914,0(8992,8)$ & 18288,1 & 0,37 \\
& $(9070,02)$ & & $(6407,22)$ & 3 \\
\hline Grupo I & 11613,35 & 14462,35 & 15100,33 & 0,04 \\
& $(6006,71)$ & $(7027,82)$ & $(5028,15)$ & 5 \\
\hline :UPO II & 22299,53 & 22772,10 & 21050,89 & 056, \\
& $(8421,53)$ & $(8903,93)$ & $(6315,67)$ & 2 \\
\hline
\end{tabular}

Fonte: Autores (2021).

Houve diferença estatisticamente significante para LMAP ( $\mathrm{p}=0,018237)$ e BAPSD $(\mathrm{p}=0,012949)$, na região dos dentes 13 e 23. Na região dos dentes 15 e 25, houve diferença estatisticamente significativa na medida LBAP (P=0,0023130). Na região dos dentes 17 e 27, houve diferença estatisticamente significativa nas medidas LBAP ( $\mathrm{p}=0), \mathrm{BAPSD}(\mathrm{p}=0,000116)$ e BAPSE $(\mathrm{p}=0,000004)$.

No grupo I, houve diferença estatisticamente significativa para LSAPSD (p=0,044121), na região dos dentes 13 e 23. Na região dos dentes 15 e 25, houve diferença estatisticamente significativa na medida LBAP $(\mathrm{P}=0,003267)$. Na região dos dentes 17 e 27, houve diferença estatisticamente significativa nas medidas LBAP ( $\mathrm{p}=0,002132)$, BAPSE $(\mathrm{p}=0,005318)$.

Por fim, no grupo II, houve diferença estatisticamente significante para LBAP ( $\mathrm{p}=0,003319)$, na região dos dentes 13 e 23e na região dos dentes 17 e 27, houve diferença estatisticamente significante nas medidas LBAP (p=0,000006), BAPSD $(\mathrm{p}=0,001556)$ e BAPSE $(\mathrm{p}=0,000712)$. 
Tabela 2. Medidas lineares (mm) da cavidade nasal, na região anterior (dentes 13 e 23), média (dentes 15 e 25) e posterior (dentes 17 e 27) nos níveis superior, médio e inferior, no sentido látero-lateral, nos tempos pré-operatório (T1), após cessada a ativação do aparelho (T2) e pós-operatório de 180 dias (T3). Médias (M), desvio padrão (dp), resultado do teste Anova para medidas repetidas $(\mathbf{p})(* \mathrm{p}<0,05)$ dos grupos 1 e 2 em conjunto.

\begin{tabular}{|c|c|c|c|c|c|}
\hline & Medida & $\begin{array}{c}\text { T1 } \\
\text { M(dp) }\end{array}$ & $\begin{array}{c}\text { T2 } \\
\text { M(dp) }\end{array}$ & $\begin{array}{c}\text { T3 } \\
\text { M(dp) }\end{array}$ & $\mathbf{p}$ \\
\hline \multirow{9}{*}{ Anterior } & LBAP & $19,0(3,8)$ & $19,5(3,0)$ & $19,9(3,0)$ & 0,139929 \\
\hline & LMAP & $18,3(3,7)$ & $18,7(3,8)$ & $18,4(3,7)$ & 0,018237 \\
\hline & LSAP & $6,9(1,8)$ & $6,9(1,8)$ & $6,9(1,7)$ & 0,762801 \\
\hline & BAPSD & $6,7(2,1)$ & $7,1(2,3)$ & $7,4(2,3)$ & 0,012949 \\
\hline & BAPSE & $6,2(2,1)$ & $6,4(2,1)$ & $6,7(2,1)$ & 0,138401 \\
\hline & LMAPSD & $4,5(1,5)$ & $4,9(1,6)$ & $4,6(1,7)$ & 0,185187 \\
\hline & LMAPSE & $5,2(2,1)$ & $5,2(2,1)$ & $4,9(2,1)$ & 0,109187 \\
\hline & LSAPSD & $2,3(0,8$ & $2,4(0,6)$ & $2,4(0,8)$ & 0,278164 \\
\hline & LSAPSE & $2,4(0,8)$ & $2,4(0,8)$ & $2,5(0,8)$ & 0,786133 \\
\hline \multirow{9}{*}{ Médio } & LBAP & $25,44(4,65)$ & $26,45(5,02)$ & $26,40(4,89)$ & 0,002313 \\
\hline & LMAP & $20,98(4,70)$ & $21,14(4,66)$ & $21,00(4,69)$ & 0,445924 \\
\hline & LSAP & $5,75(1,86)$ & $5,71(1,84)$ & $5,77(1,71)$ & 0,316115 \\
\hline & BAPSD & $10,51(2,33)$ & $11,21(3,80)$ & $11,58(3,73)$ & 0,073281 \\
\hline & BAPSE & $10,25(2,83)$ & $10,68(3,04)$ & $10,78(2,92)$ & 0,084851 \\
\hline & LMAPSD & $8,01(3,93($ & $8,08(3,76)$ & $7,95(3,60)$ & 0,787185 \\
\hline & LMAPSE & $6,90(4,22)$ & $6,82(3,88)$ & $6,73(4,18)$ & 0,674656 \\
\hline & LSAPSD & $2,00(0,99)$ & $2,02(0,97)$ & $2,07(0,87)$ & 0,599193 \\
\hline & LSAPSE & $1,88(1,13)$ & $1,99(1,14)$ & $2,00(1,08)$ & 0,234687 \\
\hline \multirow{9}{*}{ Posterior } & LBAP & $28,5(4,0)$ & $29,6(4,2)$ & $29,6(4,2)$ & 0 \\
\hline & LMAP & $23,2(3,7)$ & $23,3(3,9)$ & $23,4(3,9)$ & 0,379631 \\
\hline & LSAP & $5,9(4,4)$ & $5,9(4,5)$ & $5,9(4,3)$ & 0,940634 \\
\hline & BAPSD & $12,4(1,9)$ & $13,0(2,0)$ & $13,1(2,0)$ & 0,000116 \\
\hline & BAPSE & $12,5(2,2)$ & $13,1(2,3)$ & $13,2(2,2)$ & 0,000004 \\
\hline & LMAPSD & $9,9(2,5)$ & $9,9(2,9)$ & $9,9(3,0)$ & 0,964188 \\
\hline & LMAPSE & $10,4(2,1)$ & $10,4(2,2)$ & $10,3(2,0)$ & 0,977192 \\
\hline & LSAPSD & $1,9(1,9)$ & $2,1(2,1)$ & $2,1(1,8)$ & 0,723071 \\
\hline & LSAPSE & $1,9(2,1)$ & $2,0(1,9)$ & $2,0(1,9)$ & 0,138664 \\
\hline
\end{tabular}

Fonte: Autores (2021).

\section{Discussão}

Várias combinações de osteotomias maxilares foram propostas para a realização da EMCA. Neste estudo, apesar de não ter sido o principal objetivo, foi possível verificar a existência de influências das duas diferentes osteotomias maxilares nas alterações esqueléticas nasais e sobre o volume das vias aéreas superiores após EMCA, resultado que corrobora com estudos prévios da literatura (Pereira-Filho et al., 2014; Oliveira et al., 2016), demonstrando que as duas técnicas cirúrgicas utilizadas são eficazes.

O aumento volumétrico da cavidade nasal ocorre devido ao alargamento dos segmentos maxilares que compõem o assoalho da cavidade nasal (Byloff \& Mossaz, 2004; Baraldi et al., 2007; Deeb et al., 2010; Magnusson et al., 2012), podendo resultar em um aumento do fluxo de ar, consequentemente melhorando a respiração (Aras et al., 2010; Iwasaki et al., 2014), significante ou não a longo prazo (Baraldi et al, 2007; Gurgel et al., 2014). Os trabalhos encontrados na literatura sugerem que o aumento da região do assoalho nasal pode (Deeb et al, 2010) ou não (Gurgel et al., 2014) estar relacionado com a EMCA. A expansão do assoalho da cavidade nasal costuma ser entre 1,31 mm (Byloff \& Mossaz, 2004) a 2,63mm (Magnusson et al, 2012). 
Os resultados deste estudo mostraram aumento no volume da cavidade nasal em ambos os grupos, entretanto, com aumento mais evidente no Grupo I. Nós observamos aumento do volume nasal nos dois tempos pós-operatórios avaliados. Esse aumento foi estatisticamente significativo no grupo I, representando um aumento de $30 \%\left(3486,98 \mathrm{~mm}^{3}\right)$ ao comparar T1 e T3. Portanto, encontramos que a EMCA resulta em aumento do volume da cavidade nasal, mantido até o período de controle final (180 dias), concordo com estudos da literatura (Deeb et al, 2010; Nada et al., 2013). Entretanto, essas alterações, embora por vezes de significância estatística, parecem não ser suficientes para apresentar um efeito benéfico mensurável na respiração dos pacientes a longo prazo, apesar de existirem relatos de melhora em períodos iniciais (Berretin-Felix et al., 2006; Baraldi et al, 2007; Gurgel et al, 2014).

A osteotomia linear resultou em aumento volumétrico entre os períodos T2 e T3 de, em média 2\% $\left(472,57 \mathrm{~mm}^{3}\right)$, porém, sem significância estatística, além de recidiva no volume da cavidade nasal, de $8 \%\left(1721,21 \mathrm{~mm}^{3}\right)$ em média. $\mathrm{O}$ valor obtido em T3 foi $6 \%$ menor que o valor encontrado para T1, indicando que a ausência do degrau poderia implicar em maior recidiva. Entretanto, é necessário considerar que a estabilidade dos resultados também pode estar relacionada com a diferença no ritmo de ativação. No Grupo I, foi realizada uma ativação mais lenta $(0,5 \mathrm{~mm} /$ dia $)$ e intermitente, havendo um período menor para estabilização até o T3 quando comparado com o Grupo II com ativação mais rápida $(0,75 \mathrm{~mm} /$ dia) e ininterrupta. Os resultados observados ao avaliar o Grupo II corroboram com os descritos outros estudos (Deeb et al., 2010; Chang et al., 2016).

Ao analisar as três medidas lineares horizontais, observou-se uma média de alargamento do assoalho da cavidade nasal de $0,92 \mathrm{~mm}$ na região anterior, $0,96 \mathrm{~mm}$ na região média e $1,05 \mathrm{~mm}$ na região posterior, revelando um padrão de abertura em "V" invertido das paredes da cavidade nasal, com 14\% $(0,13 \mathrm{~mm})$ a mais de expansão na região posterior. Ao avaliar os grupos separadamente, houve diferenças importantes, sendo que o alargamento da cavidade nasal em sua porção inferior foi, em média, $113 \%(0,59 \mathrm{~mm})$ maior no Grupo II. Essa diferença pode indicar maiores ganhos na largura do assoalho nasal ao realizar-se a osteotomia linear, associada a um aumento de tensões na região do degrau zigomático no Grupo I, limitando a expansão nesse nível, o que viria a corroborar com o achado de Gonçales (2011) e Assis et al. (2013). Porém, a grande variabilidade individual e a diferença na quantidade de expansão necessária para a correção da discrepância devem ser levadas em consideração.

O grupo I apresentou aumento significativo da largura nasal (LBAP) nas porções média (15-25) e posterior (17-27), sendo esses de 0,92 e 0,98mm respectivamente. Essa expansão mais paralela observada ao avaliar a região dos pré-molares e molares corrobora com o descrito por Oliveira et al. (2016). O grupo II demonstrou aumento significativo da LBAP nas regiões anteriores (13-23), média (15-25) e posterior (17-27), com medidas de 1,65mm, 0,99mm e 1,11 mm, respectivamente. O aumento observado foi proporcionalmente maior na região anterior, indicando um padrão de expansão triangular, corroborando com estudos que apontam aumentos 71\% (1,95 mm) na região anterior (Aras et al., 2010) e 66\% (1,90 mm) na região posterior (Oliveira et al. 2016).

Resultados ainda mais evidentes puderam ser observados ao comparar a expansão obtida em cada um dos níveis horizontais entre os dois grupos, sendo que na região anterior (13 - 23), a abertura da porção basal da cavidade nasal (LBAP) foi 1,55mm maior no Grupo II, na região média, a expansão foi $9 \%(0,08 \mathrm{~mm})$ maior no Grupo II e na região posterior também se observa maior expansão no Grupo II, havendo em média 13\% $(0,13 \mathrm{~mm})$ a mais de expansão. Ao avaliar esses resultados, devem ser levadas em consideração as limitações da pesquisa. Como as medidas são avaliadas com base em uma reformatação sagital, com o posicionamento orientado por dois dentes, essas são as que mais sofrem influência das movimentações dentárias no estudo. Isso é especialmente importante na região anterior onde, em pacientes com discrepância maxilar transversa, é comum observar-se apinhamentos dentários, e estes tendem a ser corrigidos durante a expansão devido a movimentação dentária. 
Com base na análise dos resultados da pesquisa, observou-se que a EMCA resulta em aumento na largura do assoalho da cavidade nasal. Essa abertura foi, em média $0,98 \mathrm{~mm}$ quando considerado o total da amostra. No Grupo I, em que foi realizada osteotomia com degrau no pilar zigomático, esse aumento foi mais evidente e apresentou significância estatística nas medidas realizadas na região média (15 e 25) e posterior (17 e 27). Já no Grupo II, o maior aumento pode ser contemplado na região anterior (caninos).

Diversos estudos se propuseram a avaliar alterações do septo nasal nos indivíduos submetidos à EMCA, não encontrando alterações significantes do seu posicionamento (Gonçales et al., 2007; Altug-Atac et al., 2010; Reinbacher et al., 2013; Jensen \& Rodrigo Domingo, 2016; Landim et al., 2016). Neste estudo foi possível observar dados semelhantes, uma vez que em ambas as técnicas utilizadas houve pouca alteração do posicionamento do septo nasal, sendo que este se manteve centralizado, além do aumento da distância das paredes nasais de maneira proporcional à quantidade de expansão obtida na porção inferior da cavidade nasal. Quando os grupos foram analisados em conjunto, houve deslocamento significante do septo apenas entre a parede lateral da base da cavidade nasal e o septo do lado direito (BAPSD), indicando um desvio do septo para a esquerda em sua porção inferior, o desvio avaliado foi de, em média, 0,23mm. Na região posterior (17-27), houve aumento estatisticamente significante das medidas entre a parede lateral inferior da cavidade nasal e o septo bilateralmente (BAPSD e BAPSE), indicando deslocamento proporcional do septo às paredes da cavidade nasal, o aumento avaliado foi de em média, $0,70 \mathrm{~mm}$ do lado direito e $0,68 \mathrm{~mm}$ do lado esquerdo.

Assim como observado por diversos outros autores, a maior parte das regiões avaliadas mostrou deslocamento proporcional do septo às paredes da cavidade nasal, mantendo-se centralizado na cavidade nasal (Schwarz et al., 1985; Gonçales et al., 2007; Landim et al., 2016; Altug-Atac et al, 2010). Mesmo com significância estatística, a pequena magnitude dos deslocamentos laterais observados, muitas vezes na ordem de décimos de milímetros, mostra que a EMCA sem a soltura do septo nasal teve pouca influência no posicionamento dessa estrutura. Concordando com as observações de Reinbacher et al. (2013), concluímos que mesmo com o desvio em algumas regiões analisadas, estas não parecem necessariamente resultar em repercussões clínicas relevantes.

Os resultados desse estudo corroboram com os achados de Oliveira et al. (2014), que também indica a presença de uma expansão por vezes assimétrica, sendo menor no lado em que o septo permaneceu ligado à maxila. Embora os dois tipos de osteotomia tenham apresentado diferenças nos seus efeitos sobre a cavidade nasal, ambas mostraram resultados que seguiram a mesma tendência e foram congruentes, não existindo benefício clínico mensurável frente ao tratamento. Dessa forma, a opção da técnica a ser utilizada deve permanecer como opção do profissional, considerando que não existe nenhuma diferença clínica que justifique preferência entre um dos desenhos da osteotomia (Gonçales, 2011; Assis et al., 2013; Oliveira et al, 2016).

\section{Conclusão}

- Apesar do grupo I e II possuírem diferentes desenhos de osteotomia, mostraram-se eficazes na correção da deficiência transversal da maxila e apresentaram efeitos semelhantes sobre a cavidade nasal e o espaço aéreo faríngeo;

- A osteotomia linear da maxila foi associada a maior expansão na região anterior do assoalho da cavidade nasal, enquanto a osteotomia com degrau no pilar zigomático apresentou abertura mpredominantemente na região posterior;

- A EMCA produz aumento volumétrico da cavidade nasal relacionado com a expansão do seu assoalho; 
- A EMCA sem a osteotomia do septo nasal produz uma tendência ao deslocamento assimétrico do septo nasal de pequena magnitude

\section{Referências}

Albuquerque, G. C., Gonçales, A. G. B., Tieghi Neto, V., Nogueira, A. S., Assis, D. S. F. R., \& Gonçales, E. S. (2013). Complicações após expansão de maxila cirurgicamente assistida. Revista de Odontologia da UNESP (42), 20-24.

Altug-Atac, A. T., Atac, M. S., Kurt, G., \& Karasud, H. A. (2010). Changes in nasal structures following orthopaedic and surgically assisted rapid maxillary expansion. Int. J. Oral Maxillofac.Surg. (39), 129-135.

Alves, M. J., Baratieri, C., Matto,s C. T, Brunetto, D, Fontes, R. C, Santos, J. R, \& Ruellas A. C (2012). Is the airway volume being correctly analyzed? Am J Orthod Dentofacial Orthop 141(5), 657-61.

Angelieri, F., Cevidanes, L. H. S., Franchi, L., Gonçalves, J. R., Benavides, E., \& McNamara, J. A. (2013). Midpalatal suture maturation: Classification method for individual assessment before rapid maxillary expansion. Am J Orthod Dentofacial Orthop 144(5), 759- 769.

Angell, E. H. (1860). Treatment of irregularity of the permanent or adult teeth. Part 1. Dental Cosmos 1(10), 540-544.

Aras, A., Akay, M. C., Cukurova, I., et al (2010). Dimensional changes of the nasal cavity after transpalatal distraction using bone-borne distractor: An acoustic rhinometry and computed tomography evaluation. J Oral Maxillofac Surg (68),1487.

Asscherickx K, Govaerts E, Aerts J, \& Vande Vannet B (2016). Maxillary changes with boneborne surgically assisted rapid palatal expansion: A prospective study. Am J Orthod Dentofacial Orthop. 149(3), 374-83.

Assis, D. S. F. R., Ribeiro Júnior, P. D., Duarte, M. A. H., \& Gonçales, E. S. (2011). Evaluation of the mesiobucal gingival sulcus depth of the upper central incisors in patients submitted to surgically assisted maxillary expansion. Oral Maxillofac Surg (15), 79-84.

Assis, D. S. F. R., Xavier, T. A., Noritomi, P. Y., Gonçales, A. G., Ferreira, Jr O., \& de Carvalho, P. C. (2013). Finite element analysis of stress distribution in anchor teeth in surgically assisted rapid palatal expansion. Int J Oral Maxillofac Surg (42),1093-9.

Azenha, M. R. (2012). Alteração da base alar e da capacidade respiratória nasal em pacientes submetidos à expansão rápida da maxila cirurgicamente assistida. Rev Cir Traumatol Buco-Maxilo-Fac 12(4), 81-90.

Babacan, H., Sokucu, O., Doruk, C., \& Ay, S. (2006). Rapid maxillary expansion and surgically assisted rapid maxillary expansion effects on nasal volume. Angle Orthod 76(1), 66-71.

Ballanti, F., Lione, R, Baccetti, T, Franchi, L, \& Cozza, P (2010). Treatment and posttreatment skeletal effects of rapid maxillary expansion investigated with low-dose computed tomography in growing subjects. Am J Orthod Dentofacial Orthop 138(3), 311-7.

Baraldi C. E, Pretto S M, \& Puricelli E (2007). Evaluation of surgically assisted maxillary expansion using acoustic rhinometry and posteroanterior cephalometry. Int J Oral Maxillofac Surg (36),305-9.

Baratieri C, Alves M Jr, Sant'anna E. F, Nojima Mda C, \& Nojima L. I. (2011). 3D mandibular positioning after rapid maxillary expansion in Class II malocclusion. Braz Dent J 22(5), 428-34.

Bays, R. A., \& Greco, J. M. (1992). Surgically assisted rapid palatal expansion: an outpatient technique with long-term stability. J Oral Maxillofac Surg 50(2), 110-3.

Bell, W. H., \& Epker, B. N. (1976). Surgical-orthodontic expansion of the maxilla. Am. J. Orthod 70(5), 517-528.

Bel, W. H., \& Jacobs, J. D. (1979). Surgical orthodontic correction of horizontal maxillary deficiency. J. Oral Surg 37(12), 897-902.

Berretin-Felix G, Yamashita R. P, Filho H. N, Gonales E. S, Trindade A. S Jr, \& Trindade I. E (2006). Short and long-term effect of surgically assisted maxillary expansion on nasal airway size. J Craniofac Surg 17(6),1045-9.

Bertossi D, Albanese M, Malchiodi L, Procacci P, \& Nocini P. F (2007). Surgical alar base management with a personal technique: the tightening alar base suture. Arch Facial Plast Surg 9(4), 248-51.

Betts N. J, Vanarsdall R. L, Barber H. D, Higgins-Barber K, \& Fonseca R. J (1995). Diagnosis and treatment of transverse maxillary deficiency. Int J Adult Orthodon Orthognath Surg 10(2),75-96.

Betts N. J (2016). Surgically assisted maxillary expansion. Atlas Oral Maxillofac Surg Clin North Am 24(1), 67-77.

Boryor A, Geiger M, Hohmann A, Wunderlich A, Sander C, Martin Sander F, \& Sande F. G. (2008). Stress distribution and displacement analysis during an intermaxillar disjunction - a three-di- mensional FEM study of a human skull. J Biomech (41),376-382.

Brown, G V. I. (1938). The surgery of oral and facial diseases and malformation. 1938. 4th Ed., London

Koudstaal, M. J. 1., Poort, L. J., van der Wal, K. G., Wolvius, E. B., Prahl-Andersen B, \& Schulten A. J (2005). Surgically assisted rapid maxillary expansion (SARME): a review of the literature. Int J Oral Maxillofac Surg 34(7), 709-14. 
Buck L. M., Dalci O, Darendeliler M. A., \& Papadopoulou A. K (2016). Effect of Surgically Assisted Rapid Maxillary Expansion on Upper Airway Volume: A Systematic Review. J Oral Maxillofac Surg 74(5),1025-43.

Byloff F. K, \& Mossaz, C. F. Skeletal and dental changes following surgically assisted rapid palatal expansion. Eur J Orthod 26(4), 403-9.

Candido, M. S. C., Monazzi, M. S., Gabrielli, M. A. C., Spin-Neto, R., Gabrielli, M. F. R., \& Pereira-Filho, V. A. (2014). Pharyngeal airway space cephalometric evaluation in transverse maxillary deficient patient after SARME. Braz J Oral Sci (13), 288-91.

Capelozza Filho, L., \& Silva Filho, O. G. (1997). Expansão rápida da maxila: considerações gerais e aplicação clínica. Parte II. Rev Dental Press Ortodon Ortop Facial 2(4),86-108.

Chamberland S., \& Proffit W. R (2011). Short-term and long-term stability of surgically assisted rapid palatal expansion revisited. Am J Orthod Dentofacial Orthop 139(6), 815-822.

Chang Y, Koenig L. S, Pruszynski J. E, TBradley T. G, Bosio J. A, \& Liu D (2013). Dimensional changes of upper airway after rapid maxillary expansion: a prospective cone-beam computed tomography study. Am J Orthod Dentofacial Orthop 143(4), 462-470.

Chung C. H, \& Goldman A. M (2004). Dental tipping and rotation immediately after surgically assisted rapid palatal expansion. Eur J Orthod (25), 353-8.

Chung C. H, Woo A, Zagarinsky J, Vanarsdall R. L, \& Fonseca R. J (2001). Maxillary sagittal and vertical displacement induced by surgically assisted rapid palatal expansion. Am J Orthod Dentofacial Orthop 120(2), 144-8.

Compadretti G. C, Tasca I, \& Bonetti G. A (2006). Nasal airway measurements in children treated by rapid maxillary expansion. Am J Rhinol 20(4), 385-93.

Cureton S. L, \& Cuenin M (1999). Surgically assisted rapid palatal expansion: orthodonthic preparation for clinical sucess. Am J Orthod Dentofacial Orthop (116), 46-59.

De Felippe N. L. O., Bhushan N, Da Silveira A C, Viana G, \& Smith B (2009). Long-term effects of orthodontic therapy on the maxillary dental arch and nasal cavity. Am J Orthod Dentofacial Orthop 136(4), 490.e1-8.

Deeb W, Hansen L, Hotan L, Hietschold V, Harzer W, \& Tausche E (2010). Changes in nasal volume after surgically assisted bone-borne rapid maxillary expansion. Am J Orthod Dentofacial Orthop (137), 782-9.

Dergin G, Aktop S, Varol A, Ugurlu F, \& Garip H (2015). Complications related to surgically assisted rapid palatal expansion. Oral Surg Oral Med Oral Pathol Oral Radiol 119(6), 601-7.

El H, \& Palomo J. M (2010). Measuring the airway in 3 dimensions: a reliability and accuracy study. Am J Orthod Dentofacial Orthop 137(4), S50.e1-9;

Epker B. N, \& Wolford L. M (1980). Dentofacial Deformities: surgical-orthodontic correction. St. Louis: Mosby, 1980.

Garrett B. J, Caruso J. M, Rungcharassaeng K, Farrage J. R, Kim J. S, \& Taylor G. D (2008). Skeletal effects to the maxilla after rapid maxillary expansion assessed with conebeam computed tomography. Am J Orthod Dentofacial Orthop 134(1), 8-9.

Glassman A. S, Nahigian S. J, Medway J, \& Aronowitz H. I (1984). Conservative surgical orthodontic adult rapid palatal expansion: sixteen cases. Am J Orthod 86(3), 207-213.

Gonçales E. S, Assis D. R, Capelozza A. L. A., \& Alvares L. C. (2007). Estudo radiográfico digital indireto do efeito da expansão de maxila cirurgicamente assistida (EMCA) sobre o septo nasal. Rev Dental Press Ortodon Ortoped Facial (12),85-91.

Gonçales E. S, Duarte M. A, Palmieri C, Zakhary G. .M, \& Ghali G E. (2014). Retrospective analysis of the effects of orthognathic surgery on the pharyngeal airway space. J Oral Maxillofac Surg 72(11), 2227-40.

Gonçales E. S. (2011). Análise da distribuição das tensões dentárias em maxila submetida a expansão cirurgicamente assistida (tese de livre-docência). Bauru (SP): Faculdade de Odontologia de Bauru, Universidade de São Paulo.

Gonçales E. S. (2010). Cirurgia ortognática: guia de orientação para portadores de deformidades faciais esqueléticas. São Paulo: Ed Santos; 2010.

Görgülü S, Gokce S. M, Olmez H, Sagdic D, \& Ors F (2011). Nasal cavity volume changes after rapid maxillary expansion in adolescents evaluated with 3dimensional simulation and modeling programs. Am J Orthod Dentofacial Orthop (15), 633-40.

Guijarro-Martínez R, \& Swennen G. R. J (2013). Three-dimensional cone beam computed tomography definition of the anatomical subregions of the upper airway: a validation study. Int. J. Oral Maxillofac. Surg (42), 1140-1149

Gungor A. Y, \& Turkkahraman H (2009). Effects of Airway Problems on Maxillary Growth: A Review. European Journal of Dentistry 3(3), $250-254$.

Gurgel J. A, \& Tiago C. M, Normando D (2014). Transverse changes after surgically assisted rapid palatal expansion. Int J Oral Maxillofac Surg (43) 316-22.

Haas A. J. (1961). Rapid expansion of the maxillary dental arch and nasal cavity by opening the midpalatal suture. Angle Orthod 31(2), 73-90.

Hamedi Sangsari A, Sadr-Eshkevari P, Al-Dam A, Friedrich R. E, Freymiller E, \& Rashad A (2016). Surgically assisted rapid palatomaxillary expansion with or without pterygomaxillar disjunction: a systematic review and meta-analysis. J Oral Maxillofac Surg (74), 338-348.

Han U. A, Kim Y, \& Park J. U (2009). Three-dimensional finite element analysis of stress distribution and displacement of the maxilla following surgically assisted rapid maxillary expansion. J Craniomaxillofac Surg 37(3), 145-54.

Haralambidis A, Ari-Demirkaya A, Acar A, Küçükkeles N, Ates M, \& Ozkaya S (2009). Morphologic changes of the nasal cavity induced by rapid maxillary expansion: a study on 3-dimensional computed tomography models. Am J Orthod Dentofacial Orthop 136(6), 815-21. 
Holberg C, Steinhäuser S, \& Rudzki-Janson I (2007). Rapid maxillary expansion in adults:cranial stress reduction depending on the extent of surgery. Eur $J$ Orthod 29(1), 31-6.

Ilizarov G. A. (1990). Clinical applications of tension-stress effects for limb lengthening. Clin Orthop Relat Res (250), 8-26.

Iwasaki T, Takemoto Y, Inada E, Sato H, Suga H, Saitoh I, Kakuno E, Kanomi R, \& Yamasaki Y. (2014). The effect of rapid maxillary expansion on pharyngeal airway pressure during inspiration evaluated using computational fluid dynamics. International Journal of Pediatric Otorhinolaryngology (78),1258-1264.

Jafari, A, Shetty, K. S, \& Kumar M (2003).: Study of stress distribution and displacement of various craniofacial structures following application of transverse orthopedic forces: A threedimensional FEM study. Angle Orthod 73(1),12-20.

Jensen T, \& Rodrigo Domingo M (2016). Surgically assisted rapid maxillary expansion (SARME) with or without intraoperative releasing of the nasal septum. Oral Surg Oral Med Oral Pathol Oral Radiol (16), S2212-4403.

Koudstaal M. J., Poort L. J., van der Wal K. G., Wolvius E. B., Prahl-Andersen B, \& Schulten A. J. (2005). Surgically assisted rapid maxillary expansion (SARME): a review of the literature. Int J Oral Maxillofac Surg. 34(7), 709-14.

Koudstaal M. J, Smeets J. B., Kleinrensink G. J., Schulten A. J, \& van der Wal K. G. (2009). Relapse and stability of surgically assisted rapid maxillary expansion: na anatomic biomechanical study. J Oral Maxillofac Surg. 67(1), 10-4.

Kraut R. A (1984). Surgically assisted rapid maxillary expansion by opening the midpalatal suture. J Oral Maxillofac Surg (42), 651-5.

Kurt G, Altug-Atac A. T, Atac M. S., \& Karasu H. A (2010). Changes in nasopharyngeal airway following orthopedic and surgically assisted rapid maxillary expansion. J Craniofac Surg (21), 312-7.

Landim F. S, Freitas G. B., Malouf A. B, Studart L. P, Rocha N. S, de Souza Andrade E. S, Caubi A. F, Filho J. R, Oliveira E \& Silva E. D. (2011). Repercussions of surgically assisted maxillary expansion on nose width and position of septum and inferior nasal conchae. Int J Med Sci 8(8), 659-66.

Lehman Júnior J. A., Hass A. J, \& Hass D. G. (1989). Surgical orthodontic correction of transverse maxillary deficiency: a simplified aproach. Plast. Reconstr. Surg 73(1), 62-6.

Lines P. A. (2013). Adult rapid maxillary expansion with corticotomy. Am. J. Orthod. 67(1), 44-56

Magnusson, A., Bjerklin, K, Kim, H., Nilsson, P., \& Marcusson, A. (2012). Three-dimensional assessment of transverse skeletal changes after surgically assisted rapid maxillary expansion and orthodontic treatment: a prospective computerized tomography study. Am J Orthod Dentofacial Orthop (142), 825-33.

Magnusson A, Bjerklin K, Nilsson P, Jönsson F, \& Marcusson A (2011). Nasal cavity size, airway resistance, and subjective sensation after surgically assisted rapid maxillary expansion: a prospective longitudinal study. Am J OrthodDentofacial Orthop (140), 641-51.

Magnusson A, Bjerklin K, Nilsson P, \& Marcusson A (2009). Surgically assisted rapid maxillary expansion: long-term stability. Eur J Orthod. (31) 142-149.

Magnusson A (2013). Evaluation of surgically assisted rapid maxillary expansion and orthodontic treatment. Effects on dental, skeletal and nasal structures and rhinological findings. Swed Dent J Suppl (229), 1-104.

Malmström M. F. V., \& Gurgel J. Á. (2009). Avaliação da neoformação óssea na sutura palatina Marchetti C, Pironi M, Bianchi A, Musci A. Surgically assisted rapid palatal expansion vs. segmental Le Fort I osteotomy: transverse stability over a 2-year period. J Craniomaxillofac Surg (37), 74-78.

McNamara J. A Jr, Lione R, Franchi L, Angelieri F, Cevidanes L. H, Darendeliler M. A, \& Cozza P. (2015). The role of rapid maxillary expansion in the promotion of oral and general health. Prog Orthod (16), 33.

Melsen B (1972). A histological study of the influence of sutural morphology and skeletal maturation on rapid palatal expansion in children. Trans Eur Orthod Soc, 499-507.

Melson B (1975). Palatal growth studied on human autopsy material. Am J Orthod 68),42

Mitsuda S. T., Pereira M. D, Passos A. P, Hino C. T, \& Ferreira L. M (2010). Effects of surgically assisted rapid maxillary expansion on nasal dimensions using acoustic rhinometry. Oral Surg Oral Med Oral Pathol Oral Radiol Endod. (109), 191-6.

Monini S, Malagola C, Villa M. P, Tripodi C, Tarentini S, Malagnino I, Marrone V, Lazzarino A. I, \& Barbara M (2009). Rapid maxillary expansion for the treatment of nasal obstruction in children younger than 12 years. Arch Otolaryngol Head Neck Surg 135(1), 22-7.

Nada R. M, van Loon B, Schols J. G, Maal T. J, de Koning M. J, Mostafa Y. A, \& Kuijpers-Jagtman A. M (2013). Volumetric changes of the nose and nasal airway 2 years after toothborne and bone-borne surgically assisted rapid maxillary expansion. Eur J Oral Sci (121), 450.

Northway W. M, Meade Jr J. B (1997). Surgically assisted rapid maxillary expansion: a comparison of technique, response and stability. Angle Orthod (67), $309-20$.

Oliveira T. F. M, Pereira-Filho V. A, Gabrielli M. A. C, Gonçales E. S, \& Santos-Pinto A (2016). Effects of lateral osteotomy on surgically assisted rapid maxillary expansion. Int. J. Oral Maxillofac. Surg (45), 490-496.

Oliveira, A. S (2016). Avaliação das alterações volumétricas da cavidade nasal decorrentes da expansão rápida de maxila assistida cirurgicamente (Tese de dissertação). Faculdade de Odontologia de Araraquara, Universidade Estadual Paulista "Júlio de Mesquita Filho"; Araraquara (SP)

Oliveira, T. F. L (2014). Avaliação da influência do septo nasal na expansão de maxila cirurgicamente assistida por meio de tomografia computadorizada de feixe cônico. (Tese de dissertação). Faculdade de Odontologia de Bauru, Universidade São Paulo; Bauru (SP. 
Pereira-Filho V. A, Monnazzi M. S, Gabrielli M. A. C, Spin-Neto R, Watanabe E. R, \& Gimenez C. M. M (2014). Volumetric upper airway assessment in patients with transverse maxillary deficiency after surgically assisted rapid maxillary expansion. Int J Oral Maxillofac Surg 43(5), 581-6.

Persson M, \& Thilander B (1997). Palatal suture closure in man from 15 to 35 years of age. Am J Orthod (72), 42-52.

Pogrel M. A, Kaban L. B, Vargervik K, \& Baumrind S (1992). Surgically assisted rapid maxillary expansion in adults. Int J Adult Orthodon Orthognath Surg (7), 37-41.

Prado G. P, Pereira M. D, Biló J. P, Masako Ferreira L, \& Pereira M. D. (2014). Stability of surgically assisted rapid palatal expansion with and without retention analyzed by 3-dimensional imaging. Am J Orthod Dentofacial Orthop 145(5), 610-6.

Reinbacher K. E, Wallner J, Pau M, Feichtinger M, Kärcher H, Quehenberger F, \& Zemann W (2013). Surgically assisted rapid maxillary expansion: feasibility of not releasing the nasal septum. Int J Oral Maxillofac Surg 42(3), 321-5.

Ribeiro P. D Junior, Gonçales E. S, Souza P. C. U, Nary H Filho, \& Luz J. G. C. (2006). Avaliação clínica dos procedimentos de expansão cirurgicamente assistida da maxila (ECAM). Rev Dental Press Ortodon Ortop Fac 11(1), 44-59.

Salgueiro D. G, Rodrigues V. H. L. O, Tieghi Neto V, Menezes C. C, Gonçales E. S, \& Ferreira Júnior O (2015). Evaluation of opening pattern and bone neoformation at median palatal suture area in patients submitted to surgically assisted rapid maxillary expansion (SARME) through cone beam computed tomography. J Appl Oral Sci 23(4), 397-404.

Sant'Ana E, Janson M, Kuriki E. U. \& Yaedú R. Y. F. (2009). Expansão cirúrgica da maxila. R Dental Press Ortodon Ortop Facial 14(5), 92-100.

Sant'Ana L. F, Pinzan-Vercelino C. R, Gurgel J. A, \& Carvalho P. S. P. (2016). Evaluation of surgically assisted rapid maxillary expansion with and without midpalatal split. Int J Oral Maxillofac Surg 45(8), 997-1001.

Scartezini G. R., Saska S, Dantas J. F. O. C, Hochuli-Vieira E, \& Gabrielli M. A. C (2007). Maxillary surgical. Expansão cirúrgica da maxila em pacientes adultos: expansão rápida assistida cirurgicamente ou osteotomia Le Fort I segmentar? Revista da literatura. Revista de Odontologia da UNESP 36(3), 267-273.

Schwarz G. M, Trash, W. J, Byrd D. L, \& Jacobs, J. D (1985). Tomographic assesment of nasal septal changes following surgical-orthodontic rapid maxillary expansion. Am J Orthod (87), 39-45.

Seeberger R, Kater W, Davids R, \& Thiele O. C (2010). Long term effects of surgically assisted rapid maxillary expansion without performing osteotomy of the pterygoid plates. J Craniomaxillofac Surg 38(3), 175-8.

Silva Filho O. G, Valladares Neto J, \& Almeida R. R (1989). Early correction of posterior crossbite: biomechanical characteristics of the appliances. $J$ Pedodont 13(3), 195-221.

Smith T, Ghoneima A, Stewart K, Liu S, Eckert G, Halum S, \& Kula K (2012). Three-dimensional computed tomography analysis of airway volume changes after rapid maxillary expansion. Am J Orthod Dentofacial Orthop 141(5), 618-626.

Tausche E, Hansen L, Hietschold V, Lagravère M. O, \& Harzer W (2007). Three-dimensional evaluation of surgically assisted implant bone-borne rapid maxillary expansion: a pilot study. Am J Orthod Dentofacial Orthop 131(4 1), 92-9.

Tecco S, Festa F, Tete S, Longhi V, \& D'Attilio M (2005). Changes in head posture after rapid maxillary expansion in mouth-breathing girls: a controlled study. Angle Orthod (75), 171-6.

Timms D. J (1999). The dawn of rapid maxillary expansion. Angle Orthod 69(3), 247-250.

Vandersea B, \& Ruvo A. T, Frost (2007). Maxillary transverse deficiency: surgical alternatives to management. Oral Maxillofac Surg Clin North Am (19), $351-68$.

Verstraaten J, Kuijpers-Jagtman A. .M, Mommaerts M. Y, Bergé S. J, Nada R. M, \& Schols J. G (2010). A systematic review of the effects of bone-borne surgical assisted rapid maxillary expansion. J Craniomaxillofac Surg 38(3), 166-74.

Vinha P. P, Faria A. C, Xavier S. P, Christino M, \& Mello Filho F. V (2016). Enlargement of the Pharynx Resulting From Surgically Assisted Rapid Maxillary Expansion. J Oral Maxillofac Surg (74), 369-379.

Wertz R. A (1968). Changes in nasal airflow incident to rapid maxillary expansion. Angle Orthod (33), 1-11.

Wreidt S, Kunkel M, Zentner A, \& Wahlmann U. W. (2001) Surgically assisted rapid palatal expansion. An acoustic rhinometric, morphometric and sonographic investigation. J Orofac Orthop 62(2), 107-15.

Zambon C. E, Ceccheti M. M, Utumi E. R, Pinna F. R, Machado G. G, Peres M. P, \& Voegels R. L (2012). Orthodontic measurements and nasal respiratory function after surgically assisted rapid maxillary expansion: an acoustic rhinometry and rhinomanometry study. Int J Oral Maxillofac Surg (41), 1120-6.

Zeng J, \& Gao X (2013). A prospective CBCT study of upper airway changes after rapid maxillary expansion. Int J Pediatr Otorhinolaryngo (77), 1805-10.

Zhao Y, Nguyen M, Gold E, Mah J. K, Sameshima G, \& Enciso R (2010). Oropharyngeal airway changes after rapid palatal expansion evaluated with conebeam computed tomography. Am J Orthod Dentofac Orthop (137), 71-8. 